\title{
Longitudinal Declines in Instrumental Activities of Daily Living in Stable and Progressive Mild Cognitive Impairment
}

\author{
Julia J. Hsiao a, b Po H. Lu ${ }^{a}$ Joshua D. Grilla Edmond Tenga, b \\ a Department of Neurology, David Geffen School of Medicine, University of California, and \\ b Veterans Affairs Greater Los Angeles Healthcare System, Los Angeles, Calif., USA
}

\section{Key Words}

Mild cognitive impairment - Dementia - Diagnosis · Activities of daily living · Functional abilities · Assessment measures

\begin{abstract}
Background: Previous cross-sectional studies suggest that assessments of instrumental activities of daily living (IADLs) may be useful for operationalizing the differences in functional deficits seen in mild cognitive impairment (MCI) and dementia. However, their utility for longitudinal changes in IADLs in the transition between $\mathrm{MCI}$ and dementia remains unclear. Methods: We analyzed longitudinal IADL data with the Functional Activities Questionnaire (FAQ) in stable (MCI-S; $n=1,318$ ) or progressive (MCI-P; $n=1,108$ ) MCI patients. Results: Larger increases in FAQ scores were seen in the MCI-P group across a 14.5-month interval, but overlapping distributions in the two groups yielded poorer discriminatory power than prior cross-sectional reports. Conclusion: Our findings emphasize the difficulties in operationalizing the criterion of 'essentially intact' IADLs in MCI, which may complicate the interpretation of disease progression in MCI treatment trials.

(C) 2014 S. Karger AG, Basel
\end{abstract}

\section{Introduction}

The transitional stage between normal cognitive aging and dementia has been termed mild cognitive impairment (MCI) [1]. The core diagnostic criteria for MCI include: (1) subjective cognitive complaints, (2) objective cognitive impairment, and (3) essentially intact 
Hsiao et al.: Longitudinal Declines in Instrumental Activities of Daily Living in Stable and Progressive Mild Cognitive Impairment

activities of daily living (ADLs) [1]. The criterion specifying 'essentially intact' ADLs is often the primary feature that distinguishes MCI from dementia, since subjective and objective cognitive impairment can be present in both diagnoses $[2,3]$ and declines in instrumental ADLs (IADLs) are more strongly associated with progression from MCI to dementia than declines in cognitive function [4].

In the most recent diagnostic criteria for dementia due to Alzheimer's disease (AD), the decision regarding whether ADLs are essentially intact is considered to be 'inherently a clinical judgment made by a skilled clinician on the basis of the individual circumstances of the patient' [2]. However, previous studies using standardized ADL measures have consistently reported poorer performance in individuals diagnosed with MCI relative to cognitively normal controls [5-14]. Since there are no explicit guidelines regarding the extent of functional impairment allowable within the definition of essentially intact ADLs required for a diagnosis of MCI [15], the use of subjective evaluations to make this determination may result in significant inter-rater variability in both clinical and research settings [16].

An alternative approach to distinguishing ADL performance between MCI and dementia has been the use of standardized ADL questionnaires. Several studies have examined crosssectional data to empirically determine the most effective cut-points on different assessments, including the Functional Activities Questionnaire (FAQ), the Bayer Activities of Daily Living Scale, and the modified Disability Assessment for Dementia scale, for separating participants diagnosed with MCI from those diagnosed with mild dementia, yielding sensitivities ranging from 80 to $83 \%$ and specificities ranging from 72 to $87 \%$ [17-19]. However, such cross-sectional comparisons may overestimate the utility of these assessment tools for making the more difficult diagnostic distinctions near the transition between MCI and dementia, since they include a range of participants at different stages of disease progression. Furthermore, cross-sectional studies are also unable to delineate the magnitude of functional decline across the interval that marks a change in diagnosis between $\mathrm{MCI}$ and dementia.

We sought to address these issues by examining longitudinal changes in FAQ scores among individuals meeting the criteria for $\mathrm{MCI}$ whose data are included in the Unified Data Set (UDS). The UDS is a large nationwide database that is maintained by the National Alzheimer's Coordinating Center (NACC) and compiled from participants evaluated through the Alzheimer's Disease Centers (ADCs) supported by the National Institute on Aging (NIA) [20]. We analyzed longitudinal FAQ data collected at approximately annual standardized assessments and compared interval changes in FAQ indices between individuals with stable $\mathrm{MCI}$ and those who progressed to dementia. These analyses allowed us to examine the validity of global FAQ cut-points for distinguishing MCI and mild dementia that were derived in a prior cross-sectional study [17] and to quantify the changes in FAQ indices associated with expert ADC clinicians' judgment regarding the transition between the essentially intact ADLs that characterize MCI and the impaired ADLs that define dementia.

\section{Methods}

\section{Research Participants}

The NACC UDS contains longitudinal data from 34 ADCs with current or prior funding from the NIA and includes participants with normal cognition, MCI, and dementia [20]. From the UDS, we identified 3,183 participants who were $\geq 50$ years of age, diagnosed as having MCI per modified Petersen criteria [1] at their initial visit, returned for at least one follow-up visit, received serial assessments with the FAQ, and whose data had been entered into the UDS by March 1, 2012. We eliminated 757 participants who reverted to normal cognition from further analyses. The remaining participants were divided into two groups based on whether their MCI diagnosis remained stable (MCI-S; $n=1,318$ ) or progressed to dementia due to AD [21] or other etiologies (MCI-P; $\mathrm{n}=1,108)$. Race/ethnicity data were dichotomized into two categories: non- 
Hsiao et al.: Longitudinal Declines in Instrumental Activities of Daily Living in Stable and Progressive Mild Cognitive Impairment

Hispanic Whites and other races/ethnicities. Diagnoses were independently determined at each ADC through clinician judgment and/or neuropsychological testing (including additional measures beyond those incorporated in the UDS) [20]. Participants meeting the criteria for MCI exhibited deficits in attention, memory, language, visuospatial, and/or executive functioning. MCI was subtyped as amnestic or nonamnestic based on the presence or absence of significant memory impairment. Both groups included participants with deficits in single or multiple cognitive domains.

\section{Functional and Cognitive Assessments}

IADL performance was assessed using the FAQ [22]. The FAQ solicits informant ratings of participants' performance on 10 separate categories of IADLs: (1) writing checks, paying bills, keeping financial records, (2) assembling tax or business records, (3) shopping alone, (4) playing a game of skill, (5) making coffee or tea, (6) preparing a balanced meal, (7) keeping track of current events, (8) attending to and understanding a television program, book, or magazine, (9) remembering appointments, family occasions, or medications, and (10) traveling out of the neighborhood. Each category is rated from 0 to 3 (0: normal; 1: has difficulty, but does by self; 2: requires assistance; 3 : dependent), with higher scores indicative of poorer performance. The activities were not rated if the participant never performed them prior to developing cognitive difficulties or if the informant had insufficient information to provide a valid response. Global FAQ indices were calculated in two ways to accommodate missing data: (1) total FAQ scores, which only include data from participants with valid responses for all items, and (2) average FAQ item scores, which include data from all participants $[5,17]$. Prior work indicates that individual ADCs do not incorporate specific cutoff points on global FAQ indices to distinguish between MCI and dementia [17]. Cognitive performance was measured using the Mini-Mental Status Examination (MMSE) [23].

\section{Statistical Analyses}

Statistical analyses were performed using SPSS 22 for Mac (IBM, Armonk, N.Y., USA). Given the variability in the overall length of follow-up for MCI participants in the UDS, we focused on the most recent longitudinal intervals included in this dataset. For the MCI-S group, we calculated the relative changes in FAQ indices between the last recorded UDS visit (visit F) and the visit that immediately preceded it (visit F-1). For the MCI-P group, we calculated the relative changes in FAQ indices between the first visit at which these participants received a diagnosis of dementia (visit F) and the two visits immediately preceding it, during which they still met the diagnostic criteria for MCI (visits F-1 and F-2). Cross-sectional data were compared between groups using unpaired t tests for continuous variables and $\chi^{2}$ tests for categorical variables. FAQ indices were compared across the MCI-S and MCI-P groups using analysis of covariance (ANCOVA) and were adjusted for demographic differences. Within-subject comparisons of global FAQ indices across visits F-2, $\mathrm{F}-1$, and $\mathrm{F}$ in the MCI-P group were performed using paired t tests. Binomial logistic regression was used to identify the changes in specific FAQ items that were most closely associated with diagnostic progression from MCI to dementia. Receiver operating characteristic (ROC) curves and sensitivity index scores ( $d$ ') were used to identify the discriminative power of and optimal cut-points for global FAQ indices associated with the diagnostic transition between MCI and dementia.

\section{Results}

\section{Demographics}

Demographic and MMSE data for the MCI-S and MCI-P groups are shown in table 1. MCI-P subjects were significantly older at visits F and F-1 (both $\mathrm{p}<0.001$ ), more likely to be nonHispanic Whites $(\mathrm{p}<0.001)$, more likely to be diagnosed with amnestic MCI at visit F-1 (p < $0.001)$, and had lower MMSE scores ( $p<0.001)$ than MCI-S subjects. There were no differences between the groups in the time interval between visits F and F-1, gender, or years of formal education. Subsequent analyses were adjusted for race as well as age, MCI subtype, and MMSE scores at visit F-1. In the MCI-P group, $86.6 \%$ of the participants progressed to a diagnosis of possible or probable AD by visit F; the remaining participants were diagnosed with other dementing etiologies. 
Table 1. Demographic and cognitive data for MCI participants who remained diagnostically stable (MCI-S) or progressed to dementia (MCI-P)

\begin{tabular}{llll}
\hline & $\begin{array}{l}\text { MCI-S } \\
(\mathrm{n}=1,318)\end{array}$ & $\begin{array}{l}\text { MCI-P } \\
(\mathrm{n}=1,108)\end{array}$ & $\begin{array}{l}t(\text { d.f. }=2,424)^{\dagger} / \\
\chi^{2}(\mathrm{~d} . \mathrm{f} .=2,426)^{\ddagger}\end{array}$ \\
\hline Age, years $\quad$ & & \\
$\quad$ Visit F-1 & $75.4(8.7)$ & $77.0(8.7)$ & $-4.64^{*}$ \\
$\quad$ Visit F & $76.6(8.7)$ & $78.2(8.7)$ & $-4.71^{*}$ \\
Months between visits F-1 and F & $14.4(5.0)$ & $14.7(5.9)$ & -1.33 \\
Months between initial visit and visit F & $27.6(15.5)$ & $23.8(13.0)$ & $6.40^{*}$ \\
Years of education & $15.1(3.1)$ & $15.2(3.1)$ & $0.89^{\dagger}$ \\
Male & $51.3 \%$ & $51.6 \%$ & 0.03 \\
Non-Hispanic White & $78.3 \%$ & $85.7 \%$ & $22.28^{*}$ \\
Amnestic MCI at visit F-1 & $81.6 \%$ & $89.6 \%$ & $30.56^{*}$ \\
MMSE score at visit F-1 & $27.3(2.3)$ & $25.8(2.7)$ & $14.49^{*, \neq}$ \\
\hline
\end{tabular}

Values in parentheses are SD. ${ }^{*} \mathrm{p}<0.05 ;{ }^{\dagger}$ d.f. $=2,418$ due to missing data for 8 participants; ${ }^{\ddagger}$ d.f. $=2,344$ due to missing data for 80 participants.

Longitudinal Changes in Functional Indices in the MCI-S and MCI-P Groups between Visits F-1 and $F$

Complete FAQ data were obtained from a greater proportion of the MCI-S group than of the MCI-P group at visits F-1 [MCI-S: 63.6\%; MCI-P: 57.4\%; $\left.\chi^{2}(2,426)=9.64 ; p=0.002\right]$ and F [MCI-S: $64.0 \%$; MCI-P: $58.5 \% ; \chi^{2}(2,426)=7.84 ; p=0.002$ ]. The average number of FAQ items with valid responses was also significantly higher in the MCI-S group at visit F-1 [MCI-S: mean 9.36, SD 1.17; MCI-P: mean 9.22, SD 1.24; $t(2,424)=2.82 ; \mathrm{p}=0.005]$ and marginally higher at visit F [MCI-S: mean 9.31, SD 1.38; MCI-P: mean 9.22, SD 1.25; $t(2,424)=2.82 ; \mathrm{p}=$ $0.094]$. Nevertheless, over $90 \%$ of the participants in each group had $\geq 8$ FAQ items with valid responses at each visit.

Figure 1a illustrates total FAQ scores at visits F-1 and F. These data include only the participants in the MCI-S $(n=699)$ and MCI-P $(n=502)$ groups with valid responses for each FAQ item at both visits. Repeated-measures ANCOVA indicated that across visits, significantly higher total FAQ scores were seen in the MCI-P group $[F(1,1,144)=102.50, \mathrm{p}<0.001]$, in non-Hispanic Whites $[F(1,1,144)=5.70, \mathrm{p}=0.017]$, and with greater age $[F(1,1,144)=10.16$, $\mathrm{p}=0.001]$ and lower MMSE scores $[F(1,1,144)=21.15, \mathrm{p}<0.001]$. There was also a significant group $\times$ visit interaction $[F(1,1,144)=76.41, \mathrm{p}<0.001]$, indicating that greater longitudinal increases in total FAQ scores were seen in the MCI-P group (mean $=6.55$, SD 7.07) relative to the MCI-S group (mean =1.01, SD 3.61). Significantly greater longitudinal increases in total FAQ scores were also seen among older participants $[F(1,1,144)=10.80, \mathrm{p}=0.001]$ and non-Hispanic Whites $[F(1,1,144)=5.70, \mathrm{p}=0.017]$.

Figure $1 \mathrm{~b}$ illustrates average FAQ item scores at visits F-1 and F and includes data for all the participants in the MCI-S and MCI-P groups. Similar to the results described above, repeated-measures ANCOVA across visits demonstrated significantly higher average FAQ item scores in the MCI-P group $[F(1,2,324)=132.21, \mathrm{p}<0.001]$, in non-Hispanic Whites $[F(1,2,324)=10.02, \mathrm{p}=0.002]$, and with greater age $[F(1,2,324)=8.63, \mathrm{p}=0.003]$ and lower MMSE scores $[F(1,2,324)=47.07, \mathrm{p}<0.001]$. There were again significant group $\times$ visit $[F(1,2,404)=164.79, \mathrm{p}<0.001]$ and age $\times$ visit $[F(1,2,404)=17.27, \mathrm{p}<0.001]$ interactions, as larger increases in average FAQ item scores were seen in the MCI-P group (mean $=0.63$, SD 0.68) than in the MCI-S group (mean $=0.10$, SD 0.38) and in older individuals. 
Dementia

and Geriatric

Cognitive Disorders

Fig. 1. Total FAQ scores (a) and average FAQ item scores (b) across visits F-1 and F in the MCIS and MCI-P groups. Error bars indicate standard error of the mean (SEM). ${ }^{*} \mathrm{p}<0.05$ versus MCI-S.

Hsiao et al.: Longitudinal Declines in Instrumental Activities of Daily Living in Stable and Progressive Mild Cognitive Impairment
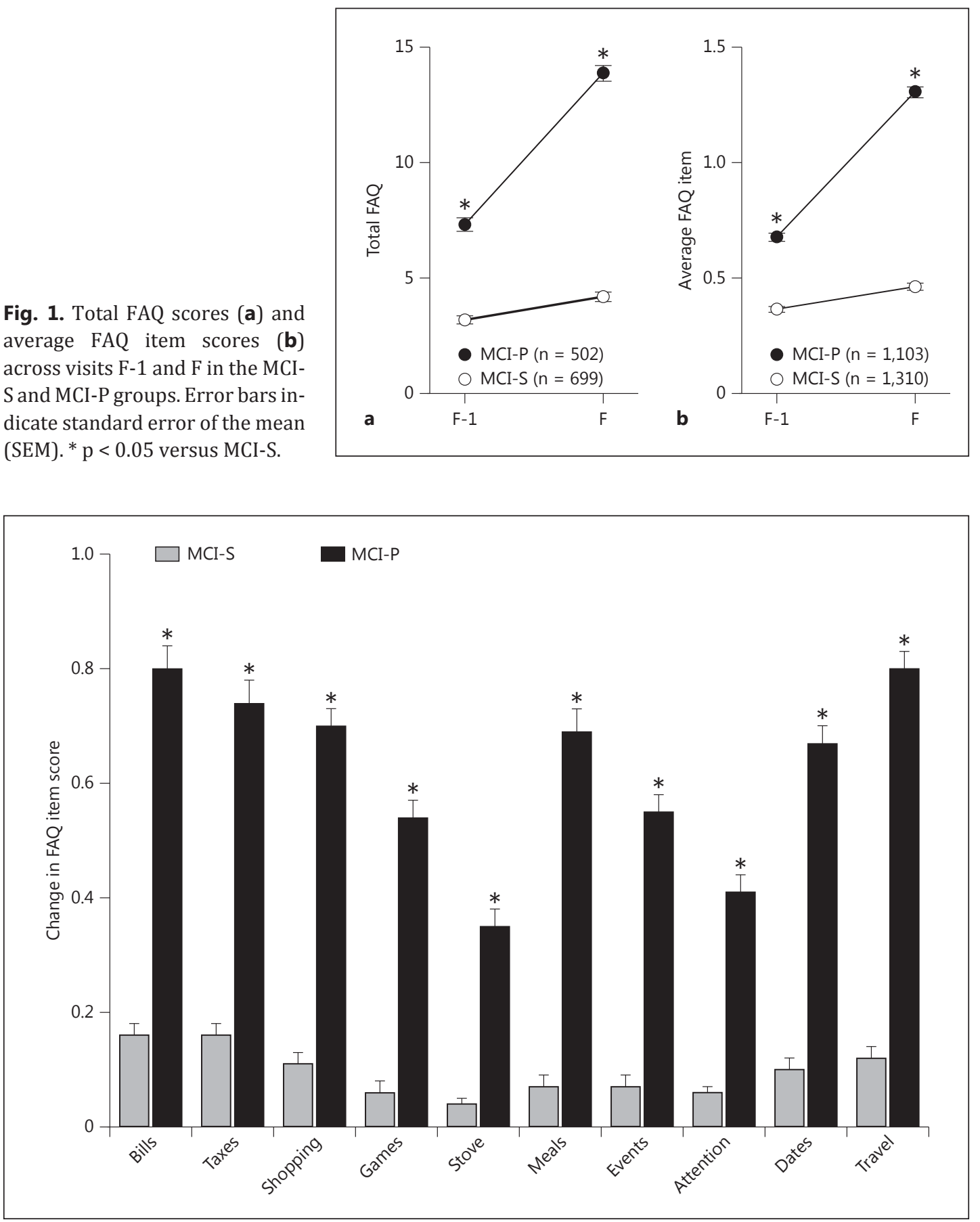

Fig. 2. Changes in individual FAQ items in the MCI-S and MCI-P groups between visits F-1 and F. Error bars indicate SEM. ${ }^{*} \mathrm{p}<0.005$ versus MCI-S.

We further investigated whether the greater longitudinal changes in global FAQ indices in the MCI-P group were preferentially driven by changes in specific individual FAQ items. However, as shown in figure 2, significantly larger increases in the MCI-P group relative to the MCI-S group were seen in all $10 \mathrm{FAQ}$ items (critical $\mathrm{p}=0.005$ after Bonferroni correction). Logistic regression analyses controlling for race, age, MCI subtype, and MMSE scores at visit 
Hsiao et al.: Longitudinal Declines in Instrumental Activities of Daily Living in Stable and Progressive Mild Cognitive Impairment

Table 2. Logistic regression analysis of demographic and functional variables associated with a diagnosis of dementia at visit $\mathrm{F}$

\begin{tabular}{|c|c|c|c|c|c|}
\hline & B & SE & Wald $\chi^{2}$ & OR (95\% CI) & $\mathrm{p}$ \\
\hline Age & 0.01 & 0.01 & 0.87 & $1.01(0.99-1.02)$ & 0.352 \\
\hline Non-Hispanic White & 0.62 & 0.19 & 10.34 & $1.86(1.28-2.72)$ & 0.001 \\
\hline MCI subtype at F-1 & 0.37 & 0.22 & 2.78 & $1.44(0.94-2.22)$ & 0.096 \\
\hline MMSE score at F-1 & -0.23 & 0.03 & 51.75 & $0.80(0.75-0.85)$ & $<0.001$ \\
\hline \multicolumn{6}{|l|}{ Change in FAQ } \\
\hline Bills & 0.32 & 0.12 & 6.88 & $1.37(1.08-1.74)$ & 0.009 \\
\hline Taxes & 0.05 & 0.11 & 0.21 & $1.05(0.85-1.31)$ & 0.650 \\
\hline Shopping & 0.31 & 0.12 & 6.50 & $1.36(1.08-1.72)$ & 0.011 \\
\hline Games & 0.27 & 0.12 & 5.16 & $1.31(1.04-1.66)$ & 0.023 \\
\hline Stove & -0.26 & 0.13 & 4.09 & $0.77(0.60-0.99)$ & 0.043 \\
\hline Meal preparation & 0.45 & 0.12 & 14.64 & $1.58(1.25-1.99)$ & $<0.001$ \\
\hline Current events & 0.22 & 0.11 & 3.62 & $1.24(0.99-1.55)$ & 0.057 \\
\hline Attention & 0.11 & 0.13 & 0.78 & $1.12(0.87-1.44)$ & 0.377 \\
\hline Remembering dates & 0.25 & 0.11 & 5.25 & $1.28(1.04-1.59)$ & 0.022 \\
\hline Traveling & 0.23 & 0.10 & 6.01 & $1.26(1.05-1.52)$ & 0.014 \\
\hline
\end{tabular}

Nagelkerke $\mathrm{R}^{2}=0.374 . \mathrm{SE}=$ Standard error; OR = odds ratio.

Table 3. Demographic data for participants in the MCI-P group with either 2 or $\geq 3$ visits

\begin{tabular}{llll}
\hline & $\begin{array}{l}\text { 2 visits } \\
(\mathrm{n}=618)\end{array}$ & $\begin{array}{l}\geq 3 \text { visits } \\
(\mathrm{n}=490)\end{array}$ & $\begin{array}{l}t(\mathrm{~d} . \mathrm{f}=1,106)^{\dagger} / \\
\chi^{2}(\mathrm{~d} . \mathrm{f} .=1,108)\end{array}$ \\
\hline Age, years & & & \\
$\quad$ Visit F-2 & $\mathrm{n} / \mathrm{a}$ & $76.6(8.5)$ & - \\
$\quad$ Visit F-1 & $76.5(8.8)$ & $77.7(8.5)$ & $-2.22^{*}$ \\
$\quad$ Visit F & $77.7(8.9)$ & $78.9(8.5)$ & $-2.43^{*}$ \\
Years of education & $15.2(3.2)$ & $15.3(3.0)$ & $-0.33^{\dagger}$ \\
Male & $50.3 \%$ & $53.3 \%$ & 0.95 \\
Non-Hispanic White & $83.6 \%$ & $88.4 \%$ & $4.96^{*}$ \\
Amnestic MCI & $88.0 \%$ & $91.6 \%$ & 3.82 \\
\hline
\end{tabular}

Values in parentheses are SD. $* \mathrm{p}<0.05 ;{ }^{\dagger}$ d.f. $=1,101$ due to missing data for 5 participants.

F-1 indicated that greater increases in difficulties with bills, shopping, games, meal preparation, remembering dates, and traveling outside the neighborhood were each independently associated with progression to dementia (table 2).

Longitudinal Changes in Functional Indices in the MCI-P Group between Visits F-2 and F-1 and Visits F-1 and F

The multiple demographic differences between the MCI-S and MCI-P groups (table 1) raised the possibility that the MCI-S group may have included more individuals at earlier stages of disease progression and/or less likely to have underlying neurodegenerative etiologies than the MCI-P group. As such, comparisons of longitudinal changes in functional indices between the MCI-S and MCI-P groups may overstate the magnitude of changes in MCI that progresses to dementia relative to stable $\mathrm{MCI}$, even after adjustment for demographic factors. Therefore, we performed additional analyses in a subset of the MCI-P group that had $\geq 3$ study 


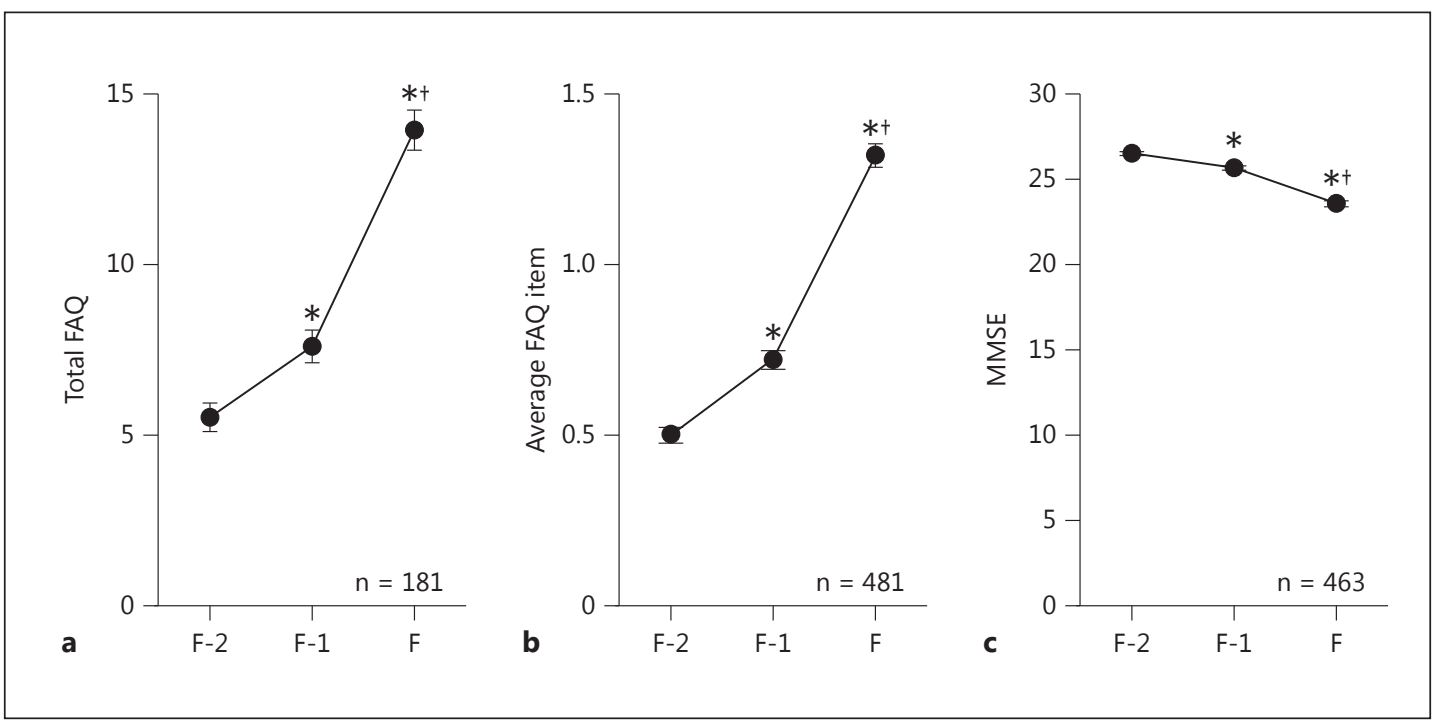

Fig. 3. Total FAQ scores (a), average FAQ item scores (b), and MMSE scores (c) in the MCI-P group across visits F-2, F-1, and F. Error bars indicate SEM. * $\mathrm{p}<0.05$ versus visit F-2; ${ }^{\dagger} \mathrm{p}<0.05$ versus visit F-1.

visits in the NACC UDS ( $\mathrm{n}=490)$. For these participants, we compared longitudinal change scores across visits F-2 and F-1, during which their MCI diagnoses remained stable, with longitudinal change scores across visits F-1 and F, during which their diagnoses progressed from MCI to dementia. These within-subject analyses incorporated smaller sample sizes but allowed for more direct investigations of the diagnostic transition from MCI to dementia without confounding demographic differences.

Demographic data for the subsets of the MCI-P participants with $\geq 3$ visits and only 2 visits are shown in table 3 . Relative to the MCI-P participants with only 2 visits, those with $\geq 3$ visits were older at visits F-1 ( $=0.015)$ and F ( $p=0.026)$, had a higher proportion of nonHispanic Whites $(p=0.026)$, and were marginally more likely to have amnestic MCI at visit $\mathrm{F}-1(\mathrm{p}=0.051)$ but had similar years of education $(\mathrm{p}=0.74)$ and gender distribution $(\mathrm{p}=$ 0.33). Global FAQ indices and MMSE scores for this subgroup are shown in figure 3. Complete FAQ data were available for $62.4 \%$ of the participants at visit F-2, for $55.9 \%$ of the participants at visit F-1, and for $60.6 \%$ of the participants at visit F. Significantly larger changes were seen across the F-1 to F interval relative to the F-2 to F-1 interval for total FAQ [F-1 to F: mean 6.36, SD 7.18; F-2 to F-1: mean 2.07, SD 5.02; $t(180)=-5.84, \mathrm{p}<0.001]$, average FAQ item [F-1 to F: mean 0.60, SD 0.66; F-2 to F-1: mean 0.21, SD 0.48; $t(480)=-9.44, p<0.001]$, and MMSE scores [F-1 to F: mean -2.11, SD 3.19; F-2 to F-1: mean -0.85 , SD 2.42; $t(462)=-6.21$, $\mathrm{p}<0.001]$. The changes in each of these indices in the MCI-P group between visits F-2 and F-1 were significantly larger than those seen in the MCI-S group between visits F-1 and $\mathrm{F}$ [total FAQ: $t(917)=3.98, \mathrm{p}<0.001$; average FAQ item: $t(1,789)=5.40, \mathrm{p}<0.001$; MMSE: $t(1,734)=3.42, \mathrm{p}<0.001]$.

Change scores for individual FAQ items in the MCI-P group across the F-2 to F-1 and F-1 to $\mathrm{F}$ intervals are shown in figure 4. After Bonferroni correction (critical $\mathrm{p}=0.005$ ), paired $\mathrm{t}$ tests demonstrated significantly greater increases across the F-1 to F interval relative to the F-2 to F-1 interval for all items except for assembling tax or business records [ $t(346)=-2.73$, $\mathrm{p}=0.007]$ and attending to and understanding a television program, book, or magazine $[t(474)=-2.74, \mathrm{p}=0.006]$. 


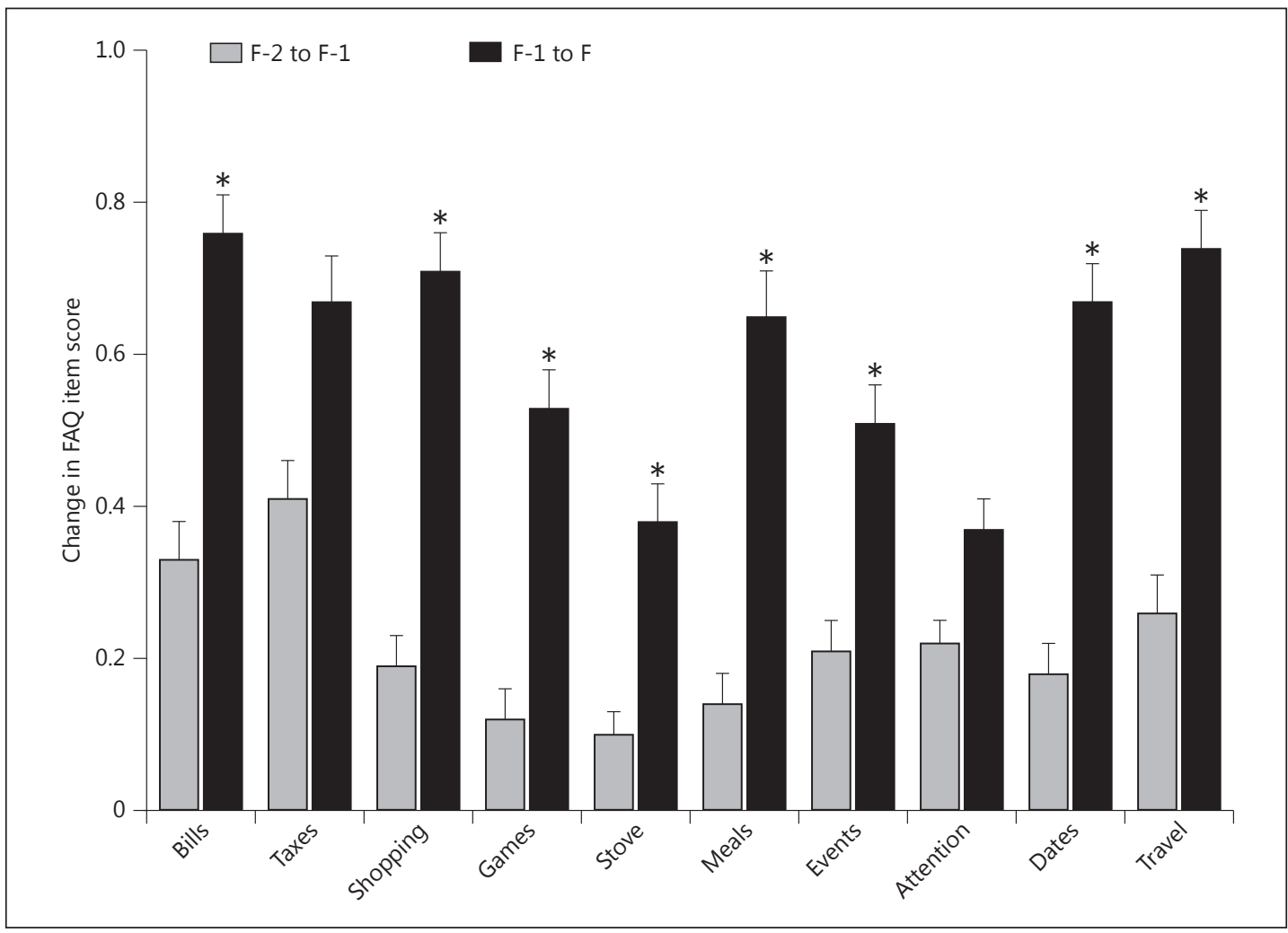

Fig. 4. Changes in individual FAQ items in the MCI-P group between visits F-2 and F-1 and between visits F-1 and F. Error bars indicate SEM. * $p<0.005$ versus F-2 to F-1 interval.

\section{Diagnostic Cut-Points Using Global FAQ Indices}

Previous analyses of cross-sectional FAQ data from the NACC UDS indicated that the optimal cut-points for distinguishing MCI from very mild AD were $<6$ for the total FAQ score (80.3\% sensitivity, $87.0 \%$ specificity, and $84.7 \%$ classification accuracy) and $<0.437$ for the average FAQ item score $(81.8 \%$ sensitivity, $77.4 \%$ specificity, and $79.1 \%$ classification accuracy) [17]. However, when we applied these cut-points to the MCI-S and MCI-P groups at visit F, their overall predictive power decreased (total FAQ score: 83.6\% sensitivity, $71.3 \%$ specificity, and $76.7 \%$ classification accuracy; average FAQ item score: $85.4 \%$ sensitivity, $61.9 \%$ specificity, and $72.6 \%$ classification accuracy) due to poorer specificity. Furthermore, when these cut-points were used to longitudinally classify the MCI-P group at visits F-1 (MCI) versus $\mathrm{F}$ (dementia), even lower specificities and classification accuracies were seen (total FAQ score: $48.8 \%$ specificity and $66.2 \%$ classification accuracy; average FAQ item score: $43.2 \%$ specificity and $64.3 \%$ classification accuracy).

ROC analyses of total FAQ scores across the MCI-S and MCI-P groups at visit F produced an area under the curve (AUC) of 0.851 [95\% confidence interval (CI): 0.832-0.871, p < 0.001] and $d^{\prime}$ of 1.43 . The optimal cut-point in this cohort was between 8 and 9 , which yielded $74.8 \%$ sensitivity, $82.1 \%$ specificity, and $79.0 \%$ classification accuracy. When this cut-point was used to distinguish total FAQ scores in the MCI-P group at visits F-1 and F, specificity dropped to $64.1 \%$ and classification accuracy fell to $69.4 \%$. ROC analyses of average FAQ item scores across the MCI-S and MCI-P groups at visit F produced an AUC of 0.826 (95\% CI: 0.809-0.842, $\mathrm{p}<0.001$ ] and $d^{\prime}$ of 1.28 . The optimal cut-point was between 0.731 and 0.732 , which yielded $73.9 \%$ sensitivity, $76.6 \%$ specificity, and $75.4 \%$ classification accuracy. When this cut-point 
was used to distinguish between average FAQ item scores in the MCI-P group at visits F-1 and F, specificity dropped to $62.3 \%$ and classification accuracy fell to $68.1 \%$.

Since the classification accuracies exhibited by cutoffs derived from global FAQ indices measured at visit $F$ in this longitudinal cohort were consistently lower than those from prior cross-sectional analyses [17], we subsequently examined the performance of cutoffs derived from the magnitude of the changes in global FAQ indices between the F-1 and F assessments. ROC analyses of changes in total FAQ scores in the MCI-S and MCI-P groups between visits F-1 and F produced an AUC of 0.780 (95\% CI: 0.752-0.809, p < 0.001] and $d^{\prime}$ of 0.99 . The optimal cut-point was between 2 and 3, which yielded $70.1 \%$ sensitivity, $79.3 \%$ specificity, and $75.4 \%$ classification accuracy. ROC analyses of changes in average FAQ item scores in the MCI-S and MCI-P groups between visits F- 1 and F produced an AUC of 0.769 (95\% CI: 0.749-0.789, p < 0.001 ] and $d^{\prime}$ of 0.97 . The optimal cut-point was between 0.275 and 0.276 , which yielded $68.7 \%$ sensitivity, $77.7 \%$ specificity, and $73.6 \%$ classification accuracy. When these cutpoints were used to distinguish changes in these indices within the MCI-P group from F-2 to F-1 and F-1 to F, poorer specificity (change in total FAQ: 61.9\%; change in average FAQ item: $60.7 \%$ ) and classification accuracy (change in total FAQ: 66.6\%; change in average FAQ item: $65.1 \%$ ) were again seen.

\section{Analyses of FAQ Indices in Amnestic MCI}

Interventional clinical trials in MCI have focused primarily on amnestic MCI due to its higher rates of progression to dementia relative to nonamnestic MCI [24-27]. Since our data also show a greater prevalence of amnestic MCI in the MCI-P group relative to the MCI-S group, we performed further analyses of FAQ indices limited to individuals diagnosed with amnestic MCI at visit F-1. These additional analyses produced results similar to those performed with the overall cohort (data not shown).

\section{Discussion}

Analyses of longitudinal IADL data from the NACC UDS revealed a marked decline in functional abilities across the diagnostic transition between MCI and dementia, as both total FAQ and average FAQ item scores approximately doubled in the MCI-P group across the F-1 to F interval. Over this interval, which averaged 14.5 months, increases in global FAQ indices associated with progression from MCI to dementia were three- to six-fold greater than the analogous increases seen with stable MCI. These findings are consistent with prior reports indicating that functional abilities are more impaired and decline more rapidly in progressive versus stable MCI [4, 6, 28-32].

Nevertheless, despite the magnitude of the longitudinal increases in global FAQ indices seen in the MCI-P group from visit F-1 to visit F, their predictive value for distinguishing MCI from dementia was poorer than that reported from cross-sectional data [17], yielding similar sensitivity but lower specificity when previously defined cut-points were used. These results suggest that expert ADC clinicians may accept higher levels of functional impairment for the criterion of essentially intact ADLs for MCI than previously estimated. The optimal diagnostic cut-point between MCI and dementia calculated from the current longitudinal dataset for total FAQ scores $(\geq 9)$ lies in the upper range of previously reported cut-points for distinguishing between normal cognition and dementia, which range from $\geq 5$ [33] to $\geq 12$ [34], but yielded poorer predictive value than those previous cross-sectional studies. These results reflect the inherent difficulty of identifying a standardized threshold for longitudinal changes in functional abilities across the diagnostic transition between MCI and dementia. 
The longitudinal increases in global FAQ indices seen in the transition from MCI to dementia were not driven by impairments in specific IADLs. Greater interval declines were seen in the MCI-P group between visits F-1 and F in most or all of the individual FAQ items relative to both of the stable MCI comparison groups (MCI-S group from F-1 to F and MCI-P group from F-2 to F-1). Furthermore, logistic regression indicated that increases in 7 out of the 10 individual FAQ categories were independently associated with progression to dementia. While these results are consistent with prior cross-sectional analyses that indicated significantly greater impairment in all IADL categories in very mild AD relative to MCI [17], there is some overlap between our results and prior work with the Lawton and Brody scale that identified a specific set of IADL deficits that were most closely associated with a diagnosis of dementia [35]. Taken together, these findings suggest that progression from MCI to dementia is associated with declining performance across a broad range of different IADLs rather than targeted declines in specific tasks. Nevertheless, given the variable difficulty and floor/ceiling effects of different IADLs, some IADL categories may be more closely associated with or predictive of diagnostic progression than others [14, 17, 35].

Our analyses indicated that poorer IADL performance in both the MCI-S and MCI-P groups was seen in the participants who were older and had lower MMSE scores. These findings are consistent with prior reports $[14,36,37]$ and support the hypothesis that individuals that are closer to the diagnostic transition from MCI to dementia (i.e. with more advanced underlying neurodegenerative disease) are more likely to have poorer functional abilities [4, 6, 28-32]. Our data also showed that non-Hispanic White participants had significantly greater IADL impairments than minority participants. This finding conflicts with previous epidemiological studies of elderly individuals, which have identified greater IADL deficits in ethnic minorities than in non-Hispanic Whites [36, 38]. However, minority participants may be more likely than non-Hispanic Whites to be diagnosed with MCI [39], particularly nonamnestic MCI [5, $40,41]$, which has been shown in some studies to be less likely to progress to dementia [2427]. Since ethnic discrepancies are larger for the prevalence of MCI than for the prevalence of dementia [42], a higher proportion of MCI diagnoses in ethnic minorities may reflect nonneurodegenerative etiologies, possibly due to cultural effects on normative data for the neuropsychological assessments, especially in non-memory domains [43-45]. Therefore, the less severe functional impairments reported in ethnic minority MCI participants in the NACC UDS database may reflect a lower likelihood of underlying neurodegenerative disease.

The limited discriminatory power of global FAQ indices for distinguishing between MCI and dementia in this longitudinal cohort can be interpreted in a number of ways. One view is that questionnaires assessing IADL function may have little value for defining the threshold of functional impairment needed for a diagnosis of dementia [46, 47], leaving open the possibility that performance-based IADL assessment tools may have greater utility for this distinction $[48,49]$. However, it is also possible that other IADL questionnaires with greater precision than the FAQ may have greater utility for this purpose. Alternatively, clinician judgment, the current gold standard for assessing IADL impairment [2] used across ADCs [17] may be variable enough between expert raters, particularly in longitudinal assessments, to limit its correlation with more formal IADL ratings, thus reducing their diagnostic accuracy. This interpretation becomes particularly problematic when clinical trials of potential therapeutic interventions for MCI are considered. Some studies have used the time to progression to dementia as their primary outcome measure and appear to have incorporated subjective clinician judgment of IADL performance into their dementia diagnoses [50]. The potential additional variability associated with using clinician judgment for determining functional impairment may further contribute to the difficulties associated with assessing potential treatments for MCI. 
Hsiao et al.: Longitudinal Declines in Instrumental Activities of Daily Living in Stable and Progressive Mild Cognitive Impairment

This study analyzed longitudinal FAQ data from a large number of participants diagnosed with MCI by expert clinicians at multiple ADCs across the United States, allowing for IADL assessments and clinical diagnoses across a wide range of geographic and demographic regions, thus increasing the generalizability of our findings. However, there are a number of factors that may limit the interpretation of our results. The NACC UDS is a convenience sample of participants volunteering for research at academic centers and may differ from communitybased epidemiological samples, particularly in participants' high levels of educational attainment. Diagnoses of MCI and dementia in the NACC UDS are based on clinical criteria [1, $21]$, and inter-ADC variability in the operationalization of both the cognitive and functional aspects of these criteria may have affected our results. Although the NACC UDS diagnoses represent the gold standard for the analyses that we have performed, the use of clinician judgment for assessing significant declines in IADLs necessarily inserts a subjective component to the diagnostic process. A significant proportion of the subjects in both the MCI-S and MCI-P groups had incomplete FAQ data from at least one visit. Incomplete FAQ data were more common in the MCI-P group, raising the possibility that these participants may have had fewer premorbid IADL responsibilities or that their informants may have been less knowledgeable about their abilities. Nevertheless, prior work suggests that informants provide more accurate IADL ratings than MCI subjects, who frequently underestimate their IADL deficits [6]. Although similar longitudinal patterns were seen using both global FAQ indices, total FAQ scores provided better classification accuracy than average FAQ item scores, despite the fact that the majority of the participants with incomplete data were missing data for only 1 or 2 items. These results confirm our prior report suggesting that the FAQ may have greater diagnostic utility when complete data are available [17]. However, our findings have not accounted for the possible presence of other medical comorbidities beyond cognitive impairment that may have impacted assessments of IADL performance.

Our analyses of longitudinal IADL data in individuals with baseline diagnoses of MCI reveal large increases in FAQ scores across the transition from MCI to dementia but also suggest significant overlap in the distributions of FAQ scores between these diagnoses. The poorer utility of global FAQ indices for the finer distinctions between MCI and dementia in this longitudinal dataset relative to prior cross-sectional analyses [17] is not wholly unexpected but reinforces previous concerns regarding the difficulty of operationalizing the criterion of essentially intact ADLs in MCI $[15,16]$. Future clinical trials of interventions aimed at delaying progression from MCI to dementia may require the development of more consistent and reliable determinations of the severity of IADL deficits that separates these diagnoses.

\section{Acknowledgements}

Support from the National Institute on Aging [P50 AG16570, K23 AG028727 to P.H.L. and K08 AG34628 to E.T. (jointly sponsored by NIA, AFAR, the John A. Hartford Foundation, the Atlantic Philanthropies, the Starr Foundation, and an anonymous donor)], the Alzheimer's Disease Research Centers of California, and the Sidell-Kagan Foundation is acknowledged. The NACC database is funded by NIA (U01 AG016976).

\section{References}

Petersen RC: Mild cognitive impairment as a diagnostic entity. J Intern Med 2004;256:183-194.

McKhann GM, Knopman DS, Chertkow H, Hyman BT, Jack CR Jr, Kawas CH, Klunk WE, Koroshetz WJ, Manly JJ, Mayeux R, Mohs RC, Morris JC, Rossor MN, Scheltens P, Carrillo MC, Thies B, Weintraub S, Phelps CH: The diagnosis of dementia due to Alzheimer's disease: recommendations from the National Institute on AgingAlzheimer's Association workgroups on diagnostic guidelines for Alzheimer's disease. Alzheimers Dement 2011; 7:263-269. 
Hsiao et al.: Longitudinal Declines in Instrumental Activities of Daily Living in Stable and Progressive Mild Cognitive Impairment

- 3 Albert MS, DeKosky ST, Dickson D, Dubois B, Feldman HH, Fox NC, Gamst A, Holtzman DM, Jagust WJ, Petersen RC, Snyder PJ, Carrillo MC, Thies B, Phelps CH: The diagnosis of mild cognitive impairment due to Alzheimer's disease: recommendations from the National Institute on Aging-Alzheimer's Association workgroups on diagnostic guidelines for Alzheimer's disease. Alzheimers Dement 2011;7:270-279.

4 Rozzini L, Chilovi BV, Conti M, Bertoletti E, Delrio I, Trabucchi M, Padovani A: Conversion of amnestic mild cognitive impairment to dementia of Alzheimer type is independent to memory deterioration. Int J Geriatr Psychiatry 2007;22:1217-1222.

5 Teng E, Becker BW, Woo E, Cummings JL, Lu PH: Subtle deficits in instrumental activities of daily living in subtypes of mild cognitive impairment. Dement Geriatr Cogn Disord 2010;30:189-197.

6 Tabert MH, Albert SM, Borukhova-Milov L, Camacho Y, Pelton G, Liu X, Stern Y, Devanand DP: Functional deficits in patients with mild cognitive impairment: prediction of AD. Neurology 2002;58:758-764.

7 Farias ST, Mungas D, Reed BR, Harvey D, Cahn-Weiner D, Decarli C: MCI is associated with deficits in everyday functioning. Alzheimer Dis Assoc Disord 2006;20:217-223.

-8 Jefferson AL, Byerly LK, Vanderhill S, Lambe S, Wong S, Ozonoff A, Karlawish JH: Characterization of activities of daily living in individuals with mild cognitive impairment. Am J Geriatr Psychiatry 2008;16:375-383.

-9 Kim KR, Lee KS, Cheong HK, Eom JS, Oh BH, Hong CH: Characteristic profiles of instrumental activities of daily living in different subtypes of mild cognitive impairment. Dement Geriatr Cogn Disord 2009;27:278-285.

10 Perneczky R, Pohl C, Sorg C, Hartmann J, Tosic N, Grimmer T, Heitele S, Kurz A: Impairment of activities of daily living requiring memory or complex reasoning as part of the MCI syndrome. Int J Geriatr Psychiatry 2006;21: 158-162.

11 Wadley VG, Crowe M, Marsiske M, Cook SE, Unverzagt FW, Rosenberg AL, Rexroth D: Changes in everyday function in individuals with psychometrically defined mild cognitive impairment in the Advanced Cognitive Training for Independent and Vital Elderly study. J Am Geriatr Soc 2007;55:1192-1198.

12 Reppermund S, Brodaty H, Crawford JD, Kochan NA, Draper B, Slavin MJ, Trollor JN, Sachdev PS: Impairment in instrumental activities of daily living with high cognitive demand is an early marker of mild cognitive impairment: the Sydney Memory and Ageing study. Psychol Med 2013;43:2437-2445.

-13 Yeh YC, Lin KN, Chen WT, Lin CY, Chen TB, Wang PN: Functional disability profiles in amnestic mild cognitive impairment. Dement Geriatr Cogn Disord 2011;31:225-232.

14 Brown PJ, Devanand DP, Liu X, Caccappolo E: Functional impairment in elderly patients with mild cognitive impairment and mild Alzheimer disease. Arch Gen Psychiatry 2011;68:617-626.

15 Gold DA: An examination of instrumental activities of daily living assessment in older adults and mild cognitive impairment. J Clin Exp Neuropsychol 2012;34:11-34.

-16 Han JW, Lee SB, Kim TH, Park JH, Lee JJ, Huh YS, Choi EA, Choe JY, Do YJ, Lee DY, Kim KW: Functional impairment in the diagnosis of mild cognitive impairment. Alzheimer Dis Assoc Disord 2011;25:225-229.

-17 Teng E, Becker BW, Woo E, Knopman DS, Cummings JL, Lu PH: Utility of the Functional Activities Questionnaire for distinguishing mild cognitive impairment from very mild Alzheimer disease. Alzheimer Dis Assoc Disord 2010;24:348-353.

18 Sanchez-Benavides G, Manero RM, Quinones-Ubeda S, de Sola S, Quintana M, Pena-Casanova J: Spanish version of the Bayer Activities of Daily Living scale in mild cognitive impairment and mild Alzheimer disease: discriminant and concurrent validity. Dement Geriatr Cogn Disord 2009;27:572-578.

19 de Rotrou J, Wu YH, Hugonot-Diener L, Thomas-Anterion C, Vidal JS, Plichart M, Rigaud AS, Hanon O: DAD-6: a 6-ltem version of the Disability Assessment for Dementia scale which may differentiate Alzheimer's disease and mild cognitive impairment from controls. Dement Geriatr Cogn Disord 2012;33:210-218.

20 Morris JC, Weintraub S, Chui HC, Cummings J, Decarli C, Ferris S, Foster NL, Galasko D, Graff-Radford N, Peskind ER, Beekly D, Ramos EM, Kukull WA: The Uniform Data Set (UDS): clinical and cognitive variables and descriptive data from Alzheimer Disease Centers. Alzheimer Dis Assoc Disord 2006;20:210-216.

21 McKhann G, Drachman D, Folstein M, Katzman R, Price D, Stadlan EM: Clinical diagnosis of Alzheimer's disease: report of the NINCDS-ADRDA work group under the auspices of Department of Health and Human Services task force on Alzheimer's disease. Neurology 1984;34:939-944.

22 Pfeffer RI, Kurosaki TT, Harrah CH Jr, Chance JM, Filos S: Measurement of functional activities in older adults in the community. J Gerontol 1982;37:323-329.

23 Folstein MF, Folstein SE, McHugh PR: 'Mini-mental state'. A practical method for grading the cognitive state of patients for the clinician. J Psychiatr Res 1975;12:189-198.

24 Busse A, Hensel A, Guhne U, Angermeyer MC, Riedel-Heller SG: Mild cognitive impairment: long-term course of four clinical subtypes. Neurology 2006;67:2176-2185.

-25 Fischer P, Jungwirth S, Zehetmayer S, Weissgram S, Hoenigschnabl S, Gelpi E, Krampla W, Tragl KH: Conversion from subtypes of mild cognitive impairment to Alzheimer dementia. Neurology 2007;68:288-291.

-26 Rasquin SM, Lodder J, Visser PJ, Lousberg R, Verhey FR: Predictive accuracy of MCI subtypes for Alzheimer's disease and vascular dementia in subjects with mild cognitive impairment: a 2-year follow-up study. Dement Geriatr Cogn Disord 2005;19:113-119.

27 Ravaglia G, Forti P, Maioli F, Martelli M, Servadei L, Brunetti N, Pantieri G, Mariani E: Conversion of mild cognitive impairment to dementia: predictive role of mild cognitive impairment subtypes and vascular risk factors. Dement Geriatr Cogn Disord 2006;21:51-58. 
Hsiao et al.: Longitudinal Declines in Instrumental Activities of Daily Living in Stable and Progressive Mild Cognitive Impairment

28 Artero S, Ancelin ML, Portet F, Dupuy A, Berr C, Dartigues JF, Tzourio C, Rouaud O, Poncet M, Pasquier F, Auriacombe S, Touchon J, Ritchie K: Risk profiles for mild cognitive impairment and progression to dementia are gender specific. J Neurol Neurosurg Psychiatry 2008;79:979-984.

-29 Di Carlo A, Lamassa M, Baldereschi M, Inzitari M, Scafato E, Farchi G, Inzitari D: CIND and MCI in the Italian elderly: frequency, vascular risk factors, progression to dementia. Neurology 2007;68:1909-1916.

-30 Peres K, Chrysostome V, Fabrigoule C, Orgogozo JM, Dartigues JF, Barberger-Gateau P: Restriction in complex activities of daily living in MCI: impact on outcome. Neurology 2006;67:461-466.

31 Okonkwo OC, Alosco ML, Jerskey BA, Sweet LH, Ott BR, Tremont G: Cerebral atrophy, apolipoprotein E ع4, and rate of decline in everyday function among patients with amnestic mild cognitive impairment. Alzheimers Dement 2010;6:404-411.

-32 Chang YL, Bondi MW, McEvoy LK, Fennema-Notestine C, Salmon DP, Galasko D, Hagler DJ Jr, Dale AM: Global Clinical Dementia Rating of 0.5 in MCI masks variability related to level of function. Neurology 2011;76:652659.

-33 Castilla-Rilo J, Lopez-Arrieta J, Bermejo-Pareja F, Ruiz M, Sanchez-Sanchez F, Trincado R: Instrumental activities of daily living in the screening of dementia in population studies: a systematic review and meta-analysis. Int J Geriatr Psychiatry 2007;22:829-836.

-34 Aprahamian I, Martinelli JE, Cecato J, Yassuda MS: Screening for Alzheimer's disease among illiterate elderly: accuracy analysis for multiple instruments. J Alzheimers Dis 2011;26:221-229.

-35 Barberger-Gateau P, Commenges D, Gagnon M, Letenneur L, Sauvel C, Dartigues JF: Instrumental activities of daily living as a screening tool for cognitive impairment and dementia in elderly community dwellers. J Am Geriatr Soc 1992;40:1129-1134.

-36 Ciol MA, Shumway-Cook A, Hoffman JM, Yorkston KM, Dudgeon BJ, Chan L: Minority disparities in disability between Medicare beneficiaries. J Am Geriatr Soc 2008;56:444-453.

-37 Marshall GA, Rentz DM, Frey MT, Locascio JJ, Johnson KA, Sperling RA: Executive function and instrumental activities of daily living in mild cognitive impairment and Alzheimer's disease. Alzheimers Dement 2011;7: 300-308.

-38 Coustasse A, Bae S, Arvidson C, Singh KP, Trevino F: Disparities in ADL and IADL disabilities among elders of Hispanic subgroups in the United States: results from the National Health Interview Survey 2001-2003. Hosp Top 2009;87:15-23.

39 Lopez OL, Jagust WJ, DeKosky ST, Becker JT, Fitzpatrick A, Dulberg C, Breitner J, Lyketsos C, Jones B, Kawas C, Carlson M, Kuller LH: Prevalence and classification of mild cognitive impairment in the Cardiovascular Health Study Cognition Study: Part 1. Arch Neurol 2003;60:1385-1389.

$\checkmark 40$ He J, Farias S, Martinez O, Reed B, Mungas D, Decarli C: Differences in brain volume, hippocampal volume, cerebrovascular risk factors, and apolipoprotein $\varepsilon 4$ among mild cognitive impairment subtypes. Arch Neurol 2009;66:1393-1399.

-41 Teng E, Tingus KD, Lu PH, Cummings JL: Persistence of neuropsychological testing deficits in mild cognitive impairment. Dement Geriatr Cogn Disord 2009;28:168-178.

42 Low LF, Harrison F, Kochan NA, Draper B, Slavin MJ, Reppermund S, Sachdev PS, Brodaty H: Can mild cognitive impairment be accurately diagnosed in English speakers from linguistic minorities? Results from the Sydney Memory and Ageing study. Am J Geriatr Psychiatry 2012;20:866-877.

43 Boone KB, Victor TL, Wen J, Razani J, Ponton M: The association between neuropsychological scores and ethnicity, language, and acculturation variables in a large patient population. Arch Clin Neuropsychol 2007; 22:355-365.

44 Manly JJ, Bell-McGinty S, Tang MX, Schupf N, Stern Y, Mayeux R: Implementing diagnostic criteria and estimating frequency of mild cognitive impairment in an urban community. Arch Neurol 2005;62:1739-1746.

45 Manly JJ, Jacobs DM, Touradji P, Small SA, Stern Y: Reading level attenuates differences in neuropsychological test performance between African American and White elders. J Int Neuropsychol Soc 2002;8:341-348.

46 Hancock P, Larner AJ: The diagnosis of dementia: Diagnostic accuracy of an instrument measuring activities of daily living in a clinic-based population. Dement Geriatr Cogn Disord 2007;23:133-139.

47 Park KW, Pavlik VN, Rountree SD, Darby EJ, Doody RS: Is functional decline necessary for a diagnosis of Alzheimer's disease? Dement Geriatr Cogn Disord 2007;24:375-379.

48 Farias ST, Harrell E, Neumann C, Houtz A: The relationship between neuropsychological performance and daily functioning in individuals with Alzheimer's disease: ecological validity of neuropsychological tests. Arch Clin Neuropsychol 2003;18:655-672.

-49 Wadley VG, Okonkwo O, Crowe M, Ross-Meadows LA: Mild cognitive impairment and everyday function: evidence of reduced speed in performing instrumental activities of daily living. Am J Geriatr Psychiatry 2008; 16:416-424.

-50 Petersen RC, Thomas RG, Grundman M, Bennett D, Doody R, Ferris S, Galasko D, Jin S, Kaye J, Levey A, Pfeiffer E, Sano M, van Dyck CH, Thal LJ: Vitamin E and donepezil for the treatment of mild cognitive impairment. N Engl J Med 2005;352:2379-2388. 